\title{
Immune properties of recombinant vaccinia virus encoding CD154 (CD40L) are determined by expression of virally encoded CD40L and the presence of $\mathrm{CD40L}$ protein in viral particles
}

\author{
Michal Bereta, ${ }^{1, a}$ Joanna Bereta, ${ }^{2, a}$ Jonas Park, ${ }^{1}$ Freddy Medina, ${ }^{3}$ Heesun Kwak, ${ }^{1}$ and \\ Howard L Kaufman ${ }^{1}$
}

\author{
${ }^{1}$ Department of Surgery and Pathology, Columbia University, New York, New York, USA; ${ }^{2}$ Department of \\ Cell Biochemistry, Faculty of Biotechnology, Jagiellonian University, Krakow, Poland; and ${ }^{3}$ Department of \\ Microbiology and Immunology, Albert Einstein College of Medicine, Bronx, New York, USA.
}

\begin{abstract}
Expression of costimulatory molecules by recombinant poxviruses is a promising strategy for enhancing therapeutic vaccines. CD40-CD40L interactions are critical for conditioning dendritic cells (DC) and priming T- and B-cell immunity. We constructed a vaccinia virus expressing murine CD40L (rV-CD40L) and studied its immunomodulatory properties in vitro. Direct DC infection with control vaccinia or psoralen/UV-inactivated rV-CD40L stimulated high levels of interleukin 12 (IL-12) release. However, replication-competent $\mathrm{rV}-\mathrm{CD} 40 \mathrm{~L}$ did not stimulate IL-12 under similar conditions. We observed a high level of CD40L protein on purified viral particles and demonstrated that induction of IL-12 by nonreplicating rV-CD40L was blocked by anti-CD40 antibodies suggesting that functional CD40L on viral particles contributed to alterations in IL-12 synthesis.

Since cross-presentation of tumor-associated antigens by DC is augmented by viral infection of tumor cells, we infected MC38 murine colon carcinoma cells with rV-CD40L. Infected cells stimulated IL-12 secretion by DC and proliferation of B cells and $\mathrm{DX5}^{+}$(NK/NKT) cells through direct CD40-CD40L interaction. A subpopulation of NKT cells expressing CD40 (NK1.1 ${ }^{+}, \mathrm{CD} 3^{\text {lo }}$ ) appeared to be a major effector population responding to MC38/rV-CD40L. These results highlight the complex immune regulatory effects of rV-CD40L defined by the cumulative effects of CD40L expression, presence of CD40L protein in viral particles, and the replication potential of the virus.
\end{abstract}

Cancer Gene Therapy (2004) 11, 808-818. doi:10.1038/sj.cgt.7700762

Published online 10 September 2004

Keywords: immunotherapy; dendritic cells; vaccinia virus; costimulation; CD40L

$\mathrm{T}$ he incorporation of genes encoding costimulatory molecules into recombinant viral vectors is broadly considered as a promising method for improving vaccine effectiveness. The coordinated expression of specific antigens with costimulatory molecules may provide a mechanism for breaking tolerance against weakly immunogenic tumor-associated antigens (TAA), which is particularly important in tumor immunotherapy. ${ }^{1}$ CD40/ CD40L interaction is a vital component of the immunological synapse formed between cooperating $\mathrm{T}$ - and B-cells, or $\mathrm{T}$ - and dendritic cells (DC) during antigen recognition and plays an important role in determining the quality of the resulting immune responses. ${ }^{2,3}$ Interaction of CD40L with CD40 expressed on B cells plays a primary role in the regulation of B-cell proliferation, synthesis of Ig and class

Received March 11, 2004.

Address correspondence and reprint requests to: Dr Howard L Kaufman, Herbert Irving Comprehensive Cancer Center, Columbia University, 177 Fort Washington Avenue, MHB-7SK, New York, NY 10032,USA. E-mail: hlk2003@columbia.edu

${ }^{a}$ Both authors contributed equally. switching, and generation of B-cell memory. ${ }^{4}$ The interaction of CD40L with CD40 on the surface of antigenpresenting cells (APCs) such as DC, macrophages, and B cells, activates signaling pathways involved in antigen presentation, costimulatory molecule expression, and synthesis of cytokines. ${ }^{5}$ CD40 signaling through tumor necrosis factor (TNF) receptor-associated factor 6 (TRAF6) suggests that CD40 is a major component of the crosstalk between the TNF receptor superfamily and the interleukin-1 receptor/Toll-like receptor (IL-1R/TLR) superfamily. ${ }^{6-8}$ In activated DC, these pathways are essential for NF- $\kappa$ B-dependent IL-12 synthesis, a central cytokine bridging innate and adaptive immunity. ${ }^{9}$

In addition to APCs, CD40 is constitutively expressed on a broad array of cells with high proliferative potential, including endothelium, some epithelial cells, and certain tumors. ${ }^{4}$ Experimental therapy targeting CD40 in human B-cell tumors has been suggested by the observation that CD40 crosslinking induced cell cycle arrest in lymphoma, leukemia, and myeloma cells. ${ }^{10}$ Conversely, there is potential for therapeutic activity by CD40 blockade, which might be beneficial in the treatment of chronic 
inflammatory diseases, such as multiple sclerosis, systemic lupus erythematosus, spontaneous autoimmune diabetes and atherosclerosis, as well as providing a mechanism for maintaining peripheral tolerance following allograft transplantation. ${ }^{11-13}$

In contrast to CD40, CD40L is primarily expressed by activated $\mathrm{CD}^{+}{ }^{+} \mathrm{T}$ lymphocytes, but it is also present in mast cells, basophils, and platelets. ${ }^{4}$ High levels of CD40L expression on a per cell basis are critical for receptor clustering and consequently CD40 signaling. ${ }^{14-16}$ Thus, the use of recombinant viral vectors, such as vaccinia virus (VV) engineered to express CD40L, provides a strategy for targeting CD40 activation. In fact, replication-competent recombinant $\mathrm{VV}$, replication-deficient adenoviral vectors, and adeno-associated viruses have all been used to achieve high levels of CD40L expression in infected cells both in vitro and in vivo. ${ }^{17-19}$

$\mathrm{VV}$ has been extensively studied as a recombinant vector for the expression of TAA and other immune regulatory genes. ${ }^{20,21}$ Preclinical animal models have demonstrated that immunological tolerance can be broken when TAA and costimulatory molecules are coexpressed by rVV-infected cells. ${ }^{22}$ However, the mechanism of antitumor immunity in these models has not been well established. While DC infected with recombinant VV can induce $\mathrm{CD}^{+}$T-cell responses against the virus and foreign antigens, other studies suggest that VV immunogenicity is mediated by crosspresentation of vacciniaderived antigens from apoptotic or necrotic VV-infected cells. ${ }^{23,24}$ Viral immunogenicity may also be influenced by cellular antigens that are carried by VV particles acquired from host cells in culture. ${ }^{25,26}$ This occurs due to the life cycle of VV within the cytoplasm of infected cells, where intracellular membranes are incorporated into intracellular enveloped virus (IEV) or cell membrane components are captured by the virus during assembly of extracellular enveloped virus (EEV) particles. The immunologic consequences of these "captured proteins" have not been fully elucidated in vaccination models, but could theoretically influence the quality of the immune response.

Here we have investigated the immunologic effects of a recombinant VV expressing CD40L (rV-CD40L) in an in vitro murine system. While we observed the activation of DC directly infected with control VV, as measured by IL-12 secretion, infection with rV-CD40L did not result in DC activation. The lack of stimulatory effect of rV-CD40L on DC could be reversed by prior psoralen/ UV treatment of the virus. Conversely, when replicationcompetent rV-CD40L was used to infect tumor cells, we observed the activation of DC, stimulation of B-cell and DX $5^{+}(\mathrm{NK} / \mathrm{NKT})$ cell proliferation, and IFN- $\gamma$ synthesis by $\mathrm{DX}^{+}$cells in a CD40/CD40L-dependent manner. Our data suggest that rV-CD40L-mediated overexpression of CD40L might lead predominantly to stimulation of humoral responses instead of DC-maturation-dependent priming of cellular immunity. These results highlight the importance of evaluating the immunoregulatory potential of poxviruses expressing costimulatory molecules and may have implications for the immunologic effects of such vectors as therapeutic vaccines.

\section{Materials and methods}

Mice

Female C57BL/6 mice were purchased from Charles River Laboratory (Wilmington, MA) and used at 8-12 weeks of age. Experimental protocols were approved by the Institutional Animal Care and Use Committee of Columbia University and followed the National Institute of Health guidelines for the care and use of laboratory animals.

\section{Viruses and plasmids}

The wild-type strain WR of VV was purchased from American Type Tissue Collection (Rockville, MD) (ATCC \#VR1354). pSC65 containing Escherechia coli $\beta$ galactosidase gene (LacZ) under control of the vaccinia early/late p7.5 promoter was a generous gift from Dr Bernard Moss, Bethesda, MD. Plasmid pBluescript (pBS II $\mathrm{KS}+$ ) containing full-length CD40L cDNA was a generous gift from Dr Chandan Guha (Montefiore Medical Center, Bronx, NY).

\section{Cell cultures}

MC38 murine colon adenocarcinoma cell line was a gift from Dr Nicholas Restifo (National Cancer Institute, Bethesda, MD). All other cell lines were from ATCC (Rockville, MD). Cells were cultured in DMEM supplemented with nonessential amino acids, sodium pyruvate $(1 \mathrm{mM})$, L-glutamine $(2 \mathrm{mM})$ and antibiotics (penicillin $100 \mathrm{U} / \mathrm{ml}$, streptomycin $100 \mu \mathrm{g} / \mathrm{ml}$ ) (complete DMEM), and $10 \% \mathrm{FCS}$ at $37^{\circ} \mathrm{C}$ in $5 \% \mathrm{CO}_{2}$. DCs were cultured in RPMI 1640 containing the same supplements (complete RPMI),

\section{Construction of $\mathrm{rV}-\mathrm{CD} 40 \mathrm{~L}$}

Construction and purification of rV-CD40L was performed according to Broder and $\mathrm{Earl}^{27}$ with modifications. The fragment containing full-length CD40L cDNA was isolated from $\mathrm{pBS}$ II $\mathrm{KS}+$ and subcloned into the $K p n \mathrm{I}$ restriction site of pSC65 under control of the synthetic vaccinia early/late promoter. pSC65 that contains the E. coli LacZ gene under control of a P7.5 vaccinia early/late promoter. Recombination performed in CV-1 cells and selection with BrdU (5-bromodeoxiuridine, Sigma, St Louis, MO) in $\mathrm{TK}^{-143 \mathrm{~B}}$ led to the generation of the virus with a dual cassette at the tk locus containing LacZ and CD40L genes controlled by distinct early/late promoters (rV-CD40L). Viral stocks (rV-LacZ and $\mathrm{rV}-\mathrm{CD} 40 \mathrm{~L}$ ) were propagated in HeLa cells and purified on sucrose gradient by ultracentrifugation.

\section{Infection of cells with $r V V s$ and psoralen/UV treatment}

Subconfluent MC38 in complete DMEM $+2.5 \%$ FCS or DC plated at the density of $2 \times 10^{5}$ cells $/ 200 \mu \mathrm{L}$ of medium/well in complete RPMI $+5 \%$ FCS were infected with viruses for 16 hours. rV-LacZ, rV-CD40L, virusinfected-MC38 as well as control, non-infected MC38 cells were subjected to psoralen and UV treatment. 
Briefly, viral suspensions or cells were incubated for 5 minutes with psoralen (4'-aminomethyl-hydrochloride, Calbiochem, San Diego, CA) at a concentration of $10 \mu \mathrm{g} / \mathrm{ml}$ and then exposed to long-wave UV light $(365 \mathrm{~nm})$ from a transilluminator placed at a distance of 3 in from cells for an additional 15 minutes.

\section{Generation of NIH3T3 cells stably transfected with} CD4OL CDNA (NIH/CD4OL)

The fragment containing full-length CD40L cDNA was cloned into the $K p n I$ restriction site of pCR3.1 (Invitrogen, Carlsbad, CA). NIH3T3 cells were transfected with pCR3.1/CD40L using lipofectamine (Invitrogen, Carlsbad, CA) and stably transfected cells were selected with geneticin $(\mathrm{G} 418)$ at a concentration of $2 \mathrm{mg} / \mathrm{ml}$.

\section{Generation of BM-DC}

Bone marrow DC (BM-DC) were prepared according to Inaba et al. ${ }^{28}$ with modifications. BM cells from femurs and tibiae were enriched in monocytes by plastic adherence and differentiated into DC by culturing with complete RPMI in the presence of rmGM-CSF and rmIL4 (Peprotech, Rocky Hill, NJ) both at $50 \mathrm{ng} / \mathrm{ml}$. After 3 days of culture, media was replaced with fresh complete RPMI containing the same supplements. DCs were cultured for 6 days in the presence of GM-CSF and IL-4 and the resulting population contained $67 \pm 14 \%$ CD11c-positive cells. Among the CD11c ${ }^{+}$cells $84 \pm 9 \%$ were $\mathrm{I}-\mathrm{A}^{\mathrm{b}}$-positive, $40 \pm 15 \%$ were CD86-positive, and $36 \pm 3 \%$ were CD40-positive (data not shown). At day 6 , cells were harvested and used in different experiments.

\section{Isolation of immune cells}

$\mathrm{T}$ - and B-cell-enriched fractions were isolated from murine splenocytes using the Pan T Cell Isolation Kit (Miltenyi Biotec, Auburn, CA). More than $90 \%$ of cells in T-cell populations were CD3-positive, and more than $90 \%$ of cells in B-cell population were B220-positive. NK $\left(\mathrm{DX} 5^{+}\right)$and non-NK cell populations were obtained by separation of splenocytes using the Mouse Anti-NK Cell (DX5) MicroBeads (Miltenyi Biotec, Auburn, CA) according to the manufacturer's instructions.

\section{Immune cells proliferation assay}

Splenocytes $\left(5 \times 10^{5} /\right.$ well $)$, or micro-bead-separated populations of immune cells $\left(2 \times 10^{5} /\right.$ well $)$, were incubated for 48 hours in 96-well plates in complete RPMI 1640 supplemented with $10 \%$ heat-inactivated FCS in the absence or the presence of MC38 $\left(1 \times 10^{4}\right.$ cells/well $)$ and in the absence or in the presence of DC $\left(5 \times 10^{4} /\right.$ well, $\gamma$-irradiated, $3 \mathrm{~Gy})$. MC38 cells - noninfected or infected with either $\mathrm{rV}-\mathrm{LacZ}$ or $\mathrm{rV}-\mathrm{CD} 40 \mathrm{~L}$ were subjected to psoralen and UV treatment (the cells infected with $\mathrm{rVV}$ at 5 MOI) or were $\gamma$-irradiated (400 Gy) (the cells infected with rVV at $0.025 \mathrm{MOI}$ ) prior to the assay. Suboptimal concentrations of ConA were added to splenocytes and $\mathrm{T}$ cells and a suboptimal dose of rmIL-4 $(5 \mathrm{ng} / \mathrm{ml})$ was added to B-cell-enriched fractions. Incorporation of $\left[{ }^{3} \mathrm{H}\right]$-thymidine $(1 \mu \mathrm{Ci} /$ well $)$, added for the last 16 hours of the incubation period was measured by a MicroBeta liquid scintillation counter (Wallac).

\section{FACS analysis}

Fc $\gamma$-receptors were blocked with anti-murine Fc $\gamma$ III/IIR monoclonal antibodies $(2.4 \mathrm{G} 2)$ and cells were stained according to standard procedures with phycoerythrin (PE)-conjugated anti-CD40L (MR1), PE-anti-CD40 (3/ 23), PE-anti-CD54 (3E2), FITC-anti-CD86 (GL1), PEanti-I-A $^{\mathrm{b}}$ (AF6-120.1), APC-anti-CD3e (145-2C11), PEanti-CD14 (rmC5-3), FITC-anti-NK1.1 (U5A2-13), allophycocyanin (APC)-conjugated anti-B220 (RA3-6B2), APC-anti-CD11c (HL3), and analyzed on a FACSCalibur Flow Cytometer (Becton Dickinson, San Jose, CA) using Cell-Quest software. All isotype control antibodies as well as monoclonal antibodies directed against murine antigens were from BD PharMingen (San Diego, CA).

\section{Cytokine production assay}

Measurement of cytokine levels in culture media was performed using ELISA assays for murine cytokines: IL-12 (p40), IL-12 (p70), IL-4, and IFN- $\gamma\left(\right.$ OptEIA $\left.^{\text {TM }}\right)$ all from BD PharMingen. All assays were carried out according to the manufacturer's instructions. Detection was performed using a microplate reader (Biorad) at $450 \mathrm{~nm}$.

\section{Visualization of CD4OL in viral particles}

E. coli $(\mathrm{DH} 5 \alpha)$ bacteria were used as a carrier for viral particles. Various doses of $\mathrm{rV}-\mathrm{CD} 40 \mathrm{~L}$ were incubated for 30 minutes on ice with overnight culture of DH5 $\alpha$ strain of $E$. coli at $1 \times 10^{7}$ bacteria/sample, washed and then stained with PE-anti-CD40L Ab in FACS staining buffer (PBS with $0.1 \%$ BSA and $0.1 \% \mathrm{NaN}_{3}$ ) for 30 minutes on ice. The levels of CD40L at the cell surface were analyzed on a FACSCalibur Flow Cytometer.

\section{Statistical analysis}

Statistical analysis was performed using the Student's $t$-test with $P<.05$ considered significant, unless otherwise stated.

\section{Results \\ CD40L expression in rV-CD40L-infected cells}

We established optimal conditions required for $\mathrm{rV}$ CD40L infection of DC and tumor cells leading to efficient surface expression of CD40L. Higher doses of virus were required for CD40L expression in DC than in MC38 colon carcinoma cells (Fig 1). Moreover, even at the highest viral dose used (20 MOI) only $50 \%$ of DC expressed CD40L, whereas infection of MC38 with 1 MOI was sufficient for the expression of CD40L in $95 \%$ of the cells. The density of CD40L on the cell surface, as evaluated by the mean fluorescence intensity (MFI), was also substantially higher on MC38 compared to DC (Fig 1). 


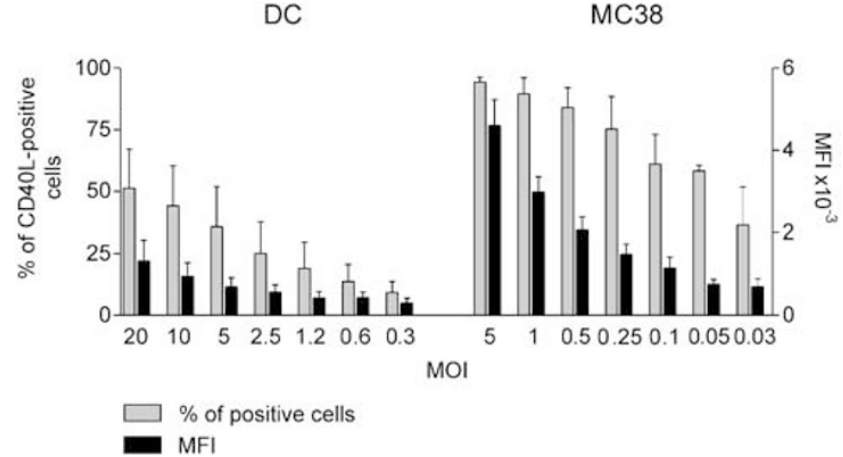

Figure 1 Flow cytometry analysis of CD40L levels on the surface of DC and MC38 infected for 16 hours with the indicated doses of rVCD40L. Data represent the average percentage and average MFI of the cells \pm SD from four independent experiments.

\section{MC38 infected with rV-CD4OL stimulates secretion of IL-12 by $D C$}

We next examined whether tumor cells infected with $\mathrm{rV}$ CD40L are able to activate DC. We chose IL-12 secretion, which is commonly used as a marker of DC activation to monitor the effects of CD40L expressed on the surface of vaccine-infected cells. CD40L expressed on stably transfected NIH3T3 cells strongly stimulated IL-12 release from DC in the absence of VV (Fig 2a). Infection of MC38 with rV-CD40L led to secretion of IL-12 p40 by coincubated DC, with the highest levels of IL-12 p40 observed at an MOI of 0.1 (Fig 2b). The decreased levels of IL-12 p40 released at higher doses of the virus are likely related to the direct cytopathic effects of VV. Consistent with this, the number of MC38 cells expressing high levels of CD40L was substantially lower 32 hours post-infection with high viral doses (Fig 2c). In contrast, the levels of CD40L expression and the number of positive cells increased considerably 32 hours after infection of MC38 with very low doses of the virus $(0.006 \mathrm{MOI})$. This explains the stimulation of IL-12 p40 secretion seen even when DC were exposed to MC38 infected at very low MOIs (Fig 2c).

The magnitude of DC activation also depends on the ratio of DC to CD40L-expressing cells. The highest levels of IL-12 p40 secretion were observed at a 1:1 ratio of DC to MC38/rV-CD40L, with comparable IL-12 p40 levels at a 4:1 ratio (Fig 2d). Stimulation of IL-12 p40 was observed up to a $64: 1$ ratio $(8.8 \mathrm{ng}$ of IL-12 p40 versus

Figure 2 Analysis of IL-12 secretion by DC incubated for 16 hours in the presence of (a) NIH/CD40L or (b, d) MC38 infected for 16 hours with rV-LacZ or rV-CD40L. (b) The ratio of DC to MC38 was 4:1. (c) Surface expression of CD40L on MC38 in 16 and 32 hours postinfection with rV-CD40L. (d) Comparison of different DC:MC38 ratios. DC $\left(2 \times 10^{5}\right)$ were incubated with decreasing numbers of MC38 cells. Data are shown as average \pm SD of (a) five independent experiments for $\mathrm{p} 40$ and two for $\mathrm{p} 70$; $(\mathbf{b}, \mathbf{c})$ duplicate samples from one experiment representative of five performed; (d) two independent experiments. ${ }^{*} P<0.02$ versus $\mathrm{rV}$-LacZ-infected cells.
$0.7 \mathrm{ng}$ of the cytokine secreted by control DC). The inhibition of MC38 cell proliferation by $\gamma$-irradiation (400 Gy), required for further experiments, did not
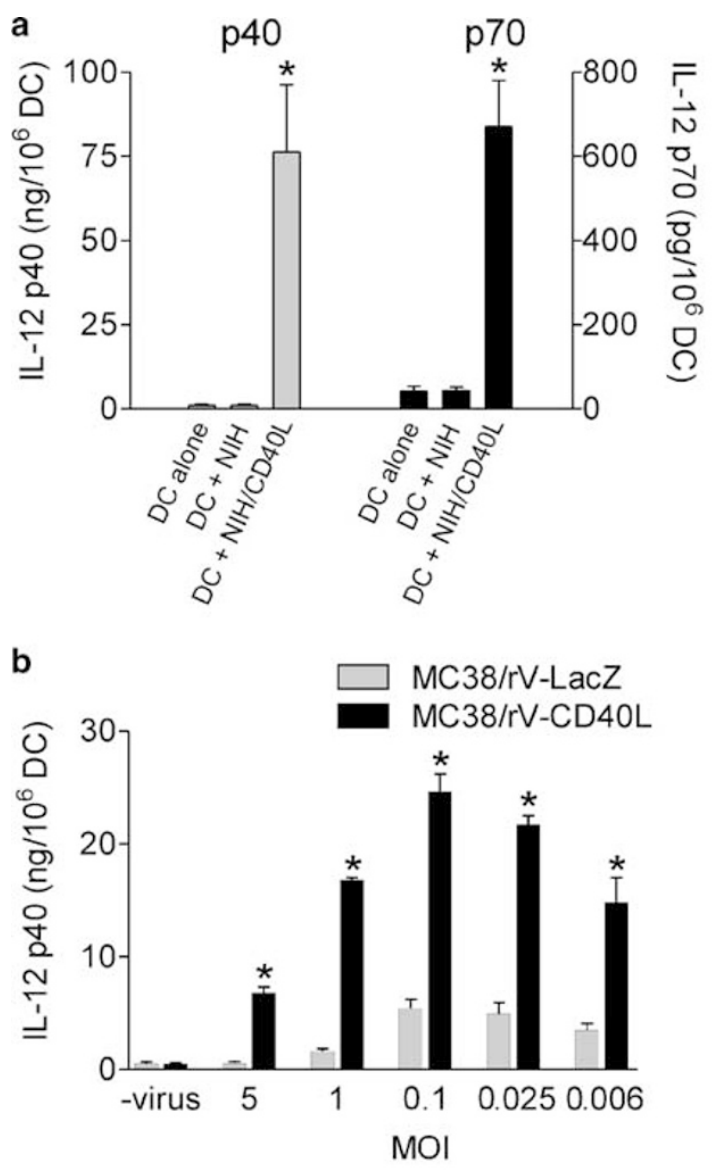

C

\begin{tabular}{|l|c|c|c|c|}
\hline \multirow{2}{*}{ MOI } & \multicolumn{2}{|c|}{ after $16 \mathrm{~h}$} & \multicolumn{2}{c|}{ after $32 \mathrm{~h}$} \\
\cline { 2 - 5 } & $\%$ cells $^{+}$ & MFI & $\%$ cells $^{+}$ & MFI \\
\hline 5 & 94 & 5574 & 72 & 1876 \\
1 & 77 & 3335 & 58 & 1952 \\
0.1 & 37 & 1416 & 58 & 1905 \\
0.025 & 12 & 1073 & 78 & 2883 \\
0.006 & 7 & 933 & 75 & 2781 \\
\hline
\end{tabular}

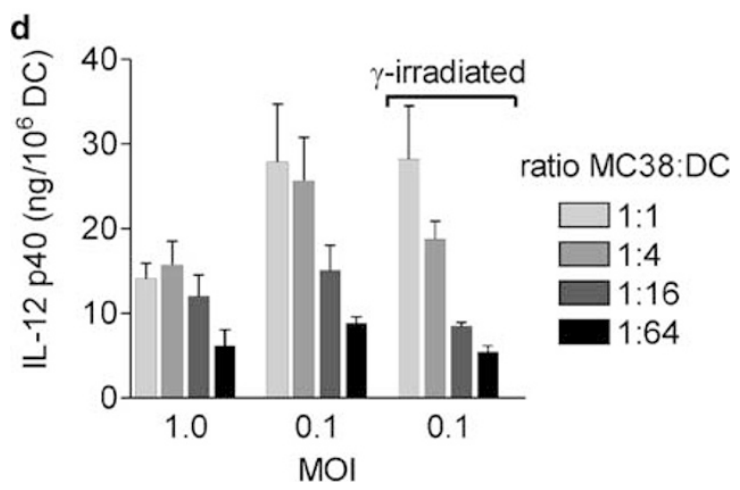


significantly affect the infectious potential of the virus (data not shown) and only slightly diminished the stimulatory effects of $\mathrm{MC} 38 / \mathrm{rV}-\mathrm{CD} 40 \mathrm{~L}$ at lower DC:MC38 ratios (Fig 2d).

\section{Psoralen/UV treatment potentiates the stimulatory effects of $r V$-CD4OL}

To limit the cytopathic effects of replication-competent VV we exposed rV-CD40L to PS/UV. However, PS/UV treatment can also inhibit transcription of some genes under control of synthetic $\mathrm{E} / \mathrm{L}$ vaccinia promoters ${ }^{29,30}$ and we found that exposure of rV-CD40L to PS/UV prior to cell infection essentially prevented expression of CD40L in the infected cells (data not shown). To avoid this problem, MC38 cells were infected with rV-CD40L for 16 hours and then treated with PS/UV, which resulted in inactivation of virus while maintaining high levels of CD40L expression (MC38/rV-CD40L ${ }^{\mathrm{N}}$ ). The effects of $\mathrm{MC} 38 / \mathrm{rV}-\mathrm{CD} 40 \mathrm{~L}^{\mathrm{N}}$ were compared to those of $\gamma$-irradiated MC38 cells carrying replication-competent virus $\left(\mathrm{MC} 38 / \mathrm{rV}-\mathrm{CD}_{40 \mathrm{~L}^{\mathrm{R}}}\right)$. Exposure of DC to both $\mathrm{MC} 38 / \mathrm{rV}$ $\mathrm{CD}_{40 \mathrm{~L}^{\mathrm{N}}}$ and $\mathrm{MC} 38 / \mathrm{rV}-\mathrm{CD} 40 \mathrm{~L}^{\mathrm{R}}$ led not only to the increased secretion of inducible IL-12-p40 (Fig 3a), but also to secretion of the biologically active $\mathrm{p} 70$ heterodimer (Fig 3b). The dose-dependence curve of IL-12 p70 secretion matched that of IL-12 p40 secretion. Since VV has strong cytopathic effects on cells in culture it is not surprising that the levels of IL-12 secretion stimulated by MC38/rV-CD40L ${ }^{\mathrm{N}}$ were higher than MC38/rV-CD40L ${ }^{\mathrm{R}}$ (Fig 3a, b). Thus, pretreatment of MC38/rV-CD40L with $\mathrm{PS} / \mathrm{UV}$ provides a mechanism for activation of DC using a replication-impaired VV vector capable of expressing CD40L. Incubation of DC with MC38/rV-LacZ did not significantly stimulate IL-12 secretion (Fig 3c) further strengthening the notion that in this experimental setting CD40L is essential for DC activation.

\section{Replication-competent $r V$-CD4OL does not stimulate} $I L-12$ release in directly infected $D C$

Next, the effect of direct DC infection with rV-CD40L on IL-12 p40 secretion was evaluated. Interestingly, DC infected with the control $\mathrm{rV}$-LacZ secreted significant levels of IL-12 p40, whereas DC infected with rV-CD40L, which differs from $\mathrm{rV}$-LacZ only by the presence of the CD40L gene, secreted negligible amounts of IL-12 p40 (Fig 4a). However, PS/UV-treated rV-CD40L stimulated IL-12 release from DC to levels comparable to that observed for DC infected with rV-LacZ (Fig 4b), suggesting that the expression of CD40L in DC might interfere with CD40-CD40L signaling pathway(s) responsible for stimulation of IL-12 release. Consistent with this hypothesis, we observed that infection of DC with $\mathrm{rV}-\mathrm{CD} 40 \mathrm{~L}$ resulted in a decrease in surface expression of CD40 that closely correlated with the expected time course of CD40L synthesis and its appearance on the cell surface 4 hours after infection (Fig 4c).

Stimulation of IL-12 secretion by DC exposed to PS/ UV-treated rV-CD40L could be blocked by anti-CD40 $\mathrm{Ab}$ (Fig 4b). Conversely, anti-CD40 Ab had no effect on

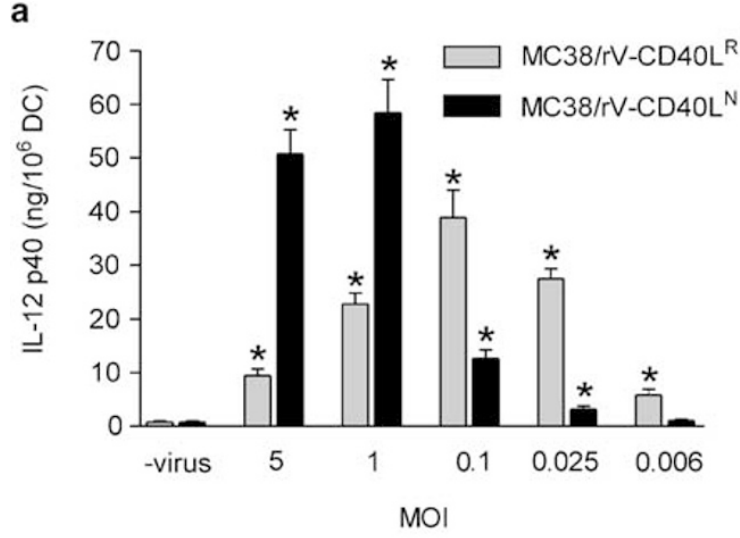

b

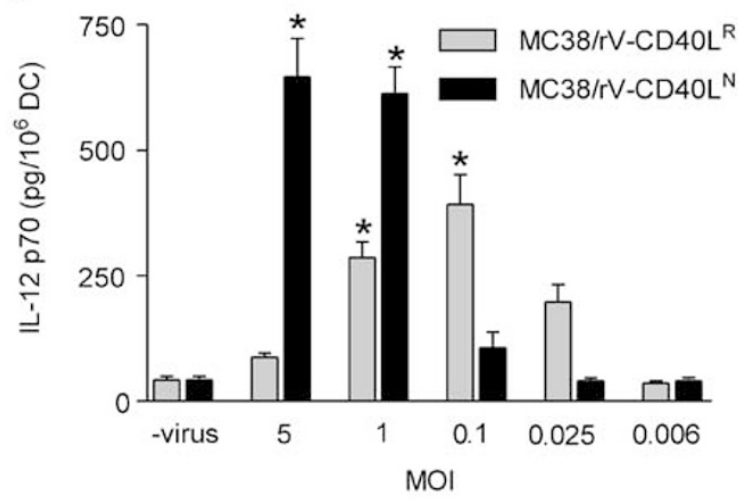

C
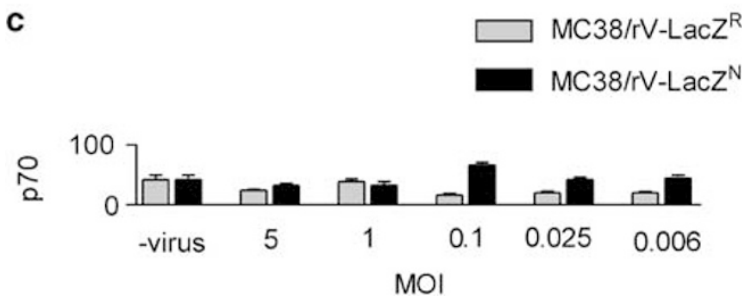

Figure 3 Analysis of IL-12 secretion by DC exposed for 16 hours to MC38 cells infected with replicating and inactivated rV-LacZ or rVCD40L. (a, b) MC38 cells were infected for 16 hours with rV-CD40L or (c) rV-LacZ, followed by $\gamma$-irradiation or PS/UV treatment and then were incubated with DC at a 1:4 ratio. " $R$ " designates replication competent virus in MC38 cells treated with $\gamma$-irradiation; "N" designates nonreplicative virus after psoralen/UV treatment of infected MC38 cells. Data represent the average \pm SD of three $(\mathbf{a}, \mathbf{c})$ and two (b) independent experiments. ${ }^{*} P<0.02$ versus control cells.

stimulation of IL-12 synthesis in DC infected with control rV-LacZ suggesting the existence of CD40-independent mechanism of stimulation of IL-12 expression. Since vaccinia particles may incorporate recombinant proteins encoded by the virus ${ }^{26}$ it is possible that particles of $\mathrm{rV}$ CD40L contained CD40L protein. In fact, increased levels of CD40L were observed on DC briefly (15 minutes) exposed to rV-CD40L (Fig 5a), but not on the cells exposed to rV-LacZ, suggesting the presence of CD40L protein on viral particles. To confirm the presence 

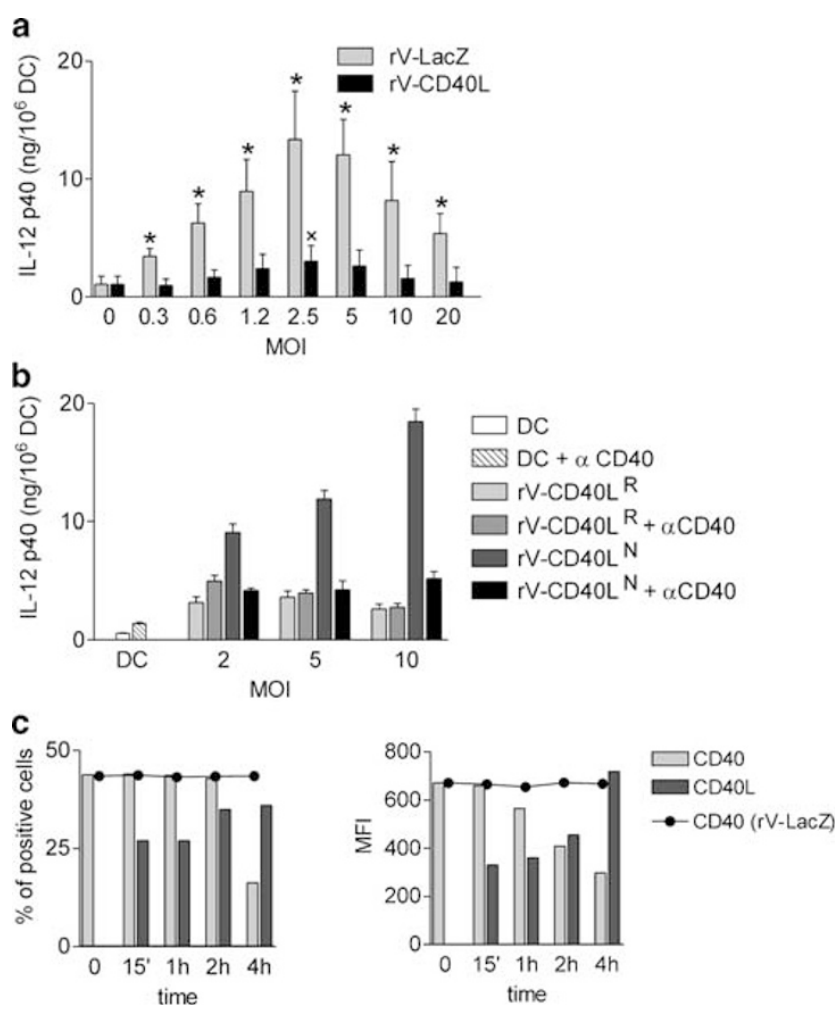

Figure 4 Analysis of IL-12 secretion by DC and surface expression of CD40 and CD40L on DC infected with rV-LacZ or rV-CD40L. (a) IL-12 p40 levels in supernatants from DC infected for 16 hours with rV-LacZ or rV-CD40L. (b) IL-12 p40 in supernatants from DC infected with replication competent $r V-C D 40 L^{R}$ or $r V-C D 40 L$ inactivated with PS/UV prior to infection of DC $\left(r V-C D 40 L^{N}\right)$ and incubated for 16 hours in the absence or in the presence of anti-CD40 Ab $(100 \mu \mathrm{g} / \mathrm{ml})$. (c) DC were infected with $\mathrm{rV}$-LacZ or with rV-CD40L (MOI 20). The levels of CD40 and CD40L were analyzed by flow cytometry. Data represent the average $\pm S D$ of (a) five independent experiments; (b) two independent experiments; (c) data are from one experiment representative of two performed. ${ }^{\star} P<0.002 ;{ }^{\times} P<0.02$ versus control cells.

of CD40L on viral particles, we used bacteria as carrier particles enabling visualization of CD40L by flow cytometry. rV-CD40L was coincubated with $E$. coli (DH $5 \alpha)$ from an overnight culture and high levels of CD40L were detected by flow cytometry (Fig 5b). A strong correlation between viral dose and the percent of bacteria stained positively for CD40L was observed (data not shown). CD40L was undetectable in control samples consisting of bacteria alone or bacteria coincubated with rV-LacZ (Fig 5b).

\section{MC38/rV-CD40L stimulates proliferation of B cells and IFN- $\gamma$ secretion by $T$ cells}

Splenocytes from naïve C57BL/6 mice were incubated for 48 hours with noninfected or infected MC38 cells, in the absence or presence of suboptimal doses of ConA, and with or without DC. MC38/rV-CD40L efficiently stimulated proliferation of splenocytes, and this effect was substantially potentiated in the presence of DC (Fig 6a). a

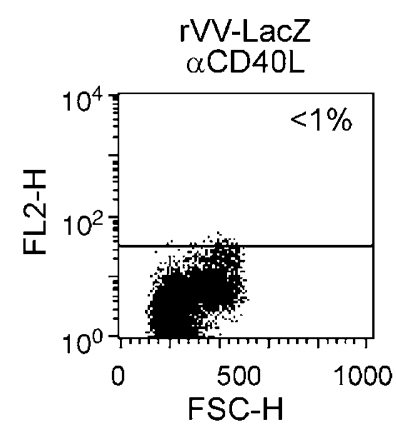

DC

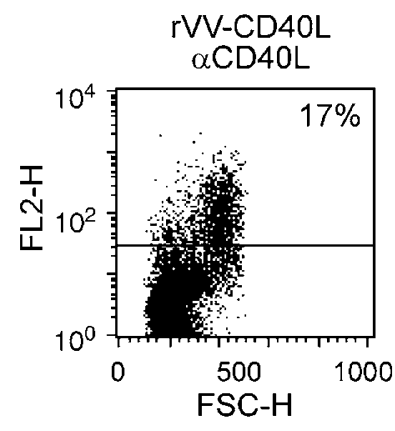

b

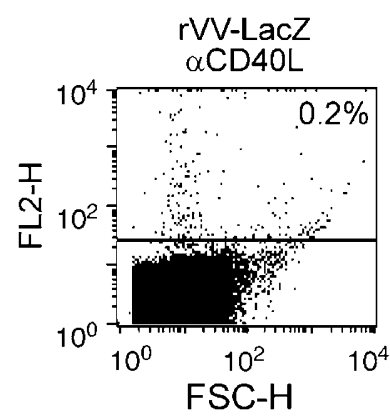

E. coli

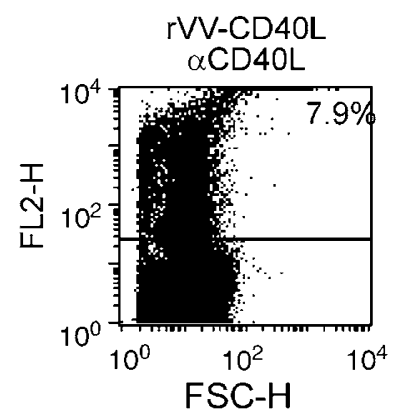

Figure 5 Detection of CD40L in rV-CD40L particles. Flow cytometric analysis of CD40L on the surface of (a) DC incubated with rV-LacZ or rV-CD40L for 15 minutes at $37^{\circ} \mathrm{C}$ at an MOI of 20. (b) E. coli incubated with rV-LacZ or rV-CD40L for 30 minutes at $4{ }^{\circ} \mathrm{C}$. Data are from a single experiment representative of three performed.

The proliferation rate was comparable between cultures containing $\mathrm{MC} 38 / \mathrm{rV}-\mathrm{CD} 40 \mathrm{~L}^{\mathrm{R}}$ and $\mathrm{MC} 38 / \mathrm{rV}-\mathrm{CD}_{40 \mathrm{~L}^{\mathrm{N}}}$ (data not shown).

To determine which populations were proliferating, enriched $\mathrm{T}$ - and B-cell fractions were separated from whole splenocytes and exposed to $\mathrm{MC} 38 / \mathrm{rV}-\mathrm{CD} 40 \mathrm{~L}$ in the presence or absence of DC. B cells proliferated in response to $\mathrm{MC} 38 / \mathrm{rV}-\mathrm{CD} 40 \mathrm{~L}$, and this effect was significantly enhanced in the presence of autologous DC (Fig 6b). In contrast to B cells, the stimulatory effect of MC38/rV-CD40L on proliferation of purified T cells was much less pronounced (Fig 6c). A slight increase in T-cell proliferation could be discerned after exposure to MC38/ rV-CD40L ${ }^{\mathrm{N}}$, and this effect appeared to be independent of ConA (Fig 6c). Addition of DC strongly potentiated ConA-induced proliferation of purified $\mathrm{T}$ cells $(>50$-fold at $200 \mathrm{ng} / \mathrm{ml}$ of ConA), which was slightly increased in the presence of $\mathrm{MC} 38 / \mathrm{rV}-\mathrm{CD} 40 \mathrm{~L}^{\mathrm{N}}$. Taken together, these experiments suggested that both $\mathrm{MC} 38 / \mathrm{rV}-\mathrm{CD}_{40 \mathrm{~L}^{\mathrm{R}}}$ and MC38/rV-CD40L ${ }^{\mathrm{N}}$ preferentially enhanced proliferation of $\mathrm{B}$ cells (Fig 6d).

In contrast to $\mathrm{MC} 38 / \mathrm{rV}-\mathrm{CD} 40 \mathrm{~L}$, neither $\mathrm{MC} 38 / \mathrm{rV}$ $\mathrm{LacZ}^{\mathrm{N}}$ nor low doses of MC38/rV-LacZ ${ }^{\mathrm{R}}(0,025 \mathrm{MOI})$ had any significant effect on proliferation of splenocytes, B cells, or T cells (Fig 6). However, exposure of immune cells to MC38 infected with relatively high doses of replication-competent $\mathrm{rV}$-LacZ $(0.5 \mathrm{MOI})$ resulted in a 
814

a
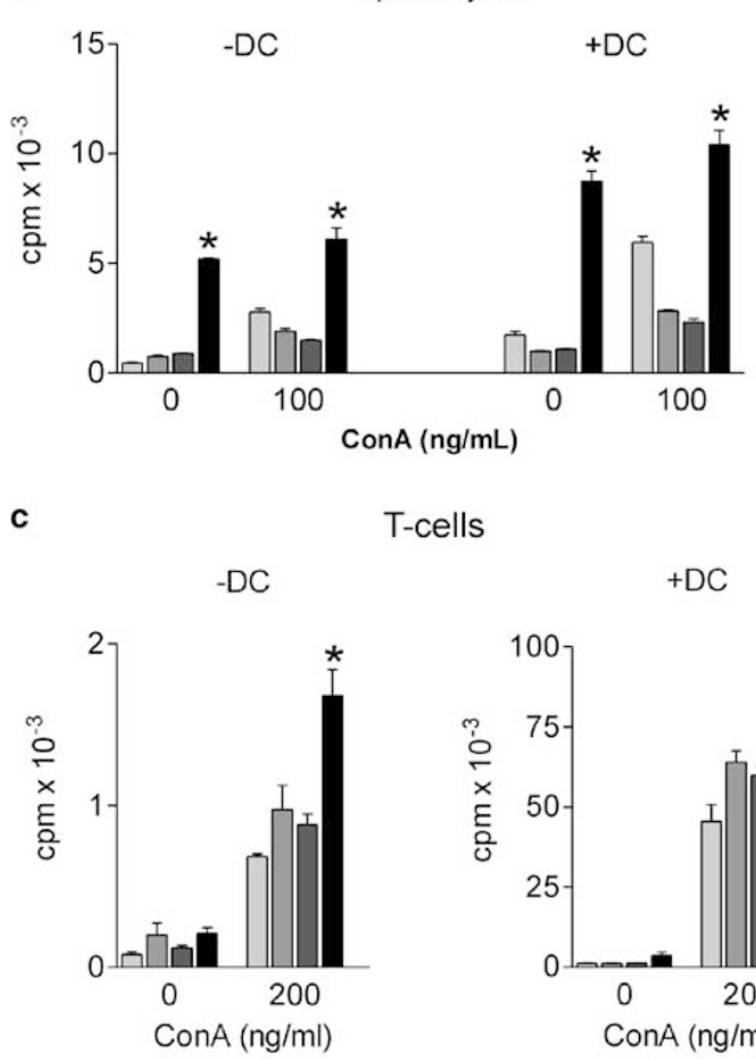

T-cells

$+\mathrm{DC}$

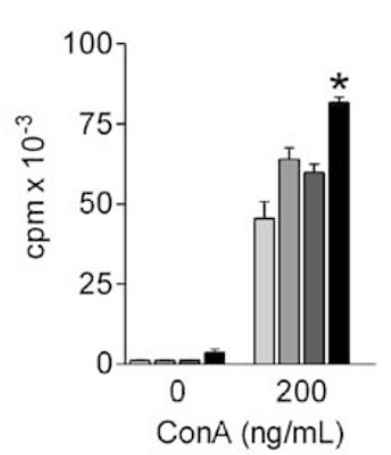

b

B-cells

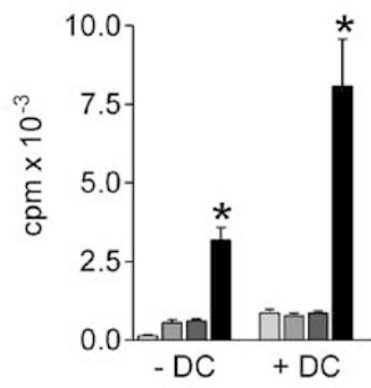

immune cells alone

$\square+\mathrm{MC} 38$

+ MC38/rV-LacZ

$+\mathrm{MC} 38 / \mathrm{rV}-\mathrm{CD} 40 \mathrm{~L}$ d
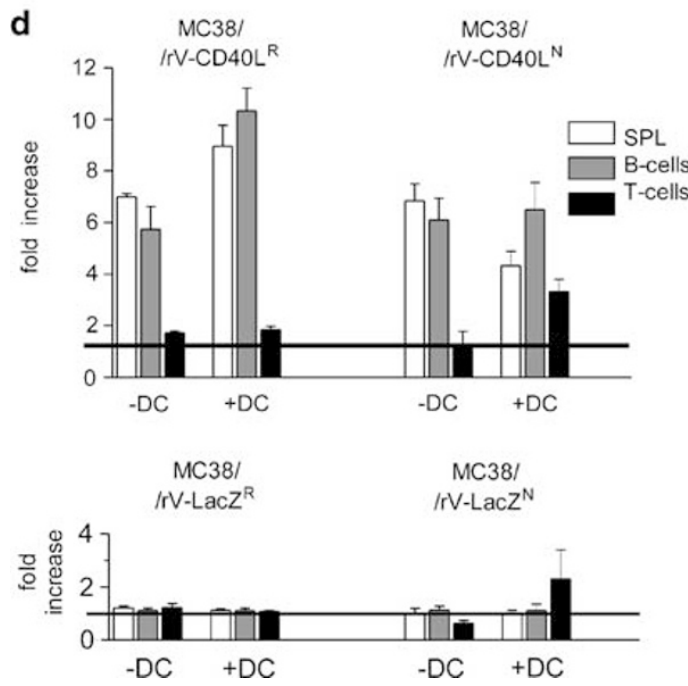

e

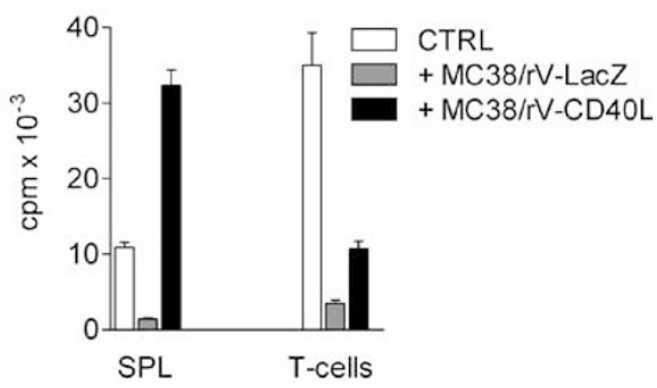

Figure $6\left[{ }^{3} \mathrm{H}\right]$-thymidine incorporation by immune cells at 48 hours after exposure to MC38 cells infected with rV-CD40L. (a) splenocytes or (b) enriched B cells incubated with control MC38, MC38/rV-LacZ ${ }^{\mathrm{R}}$, or MC38/rV-CD40L ${ }^{\mathrm{R}}$; (c) enriched T cells incubated with control MC38, MC38/rV-

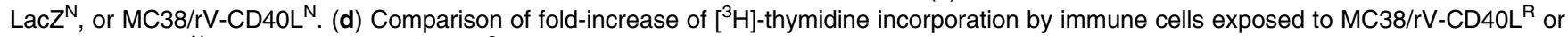
MC38/rV-CD40L ${ }^{\mathrm{N}}$ in the absence of ConA. $\left[{ }^{3} \mathrm{H}\right]$-thymidine incorporation was calibrated to background incorporation measured after exposure of immune cells to uninfected MC38 cells (control =1). (e) Comparison of the proliferation of whole splenocytes to enriched T cells co-cultured with uninfected MC38 cells (control) or with MC38 cells infected with high doses (0.5 MOI) of replication-competent rV-LacZ or rV-CD40L. Incubation was carried out in the presence of DC and suboptimal concentrations of ConA. Each value represents the average cpm \pm SD from one experiment representative of three $(\mathbf{a})$ or two $(\mathbf{b}, \mathbf{c}, \mathbf{d})$ and each determination was done in triplicate. For (e) each value represents the average $\mathrm{cpm} \pm \mathrm{SD}$ from one experiment performed in triplicate. ${ }^{*} P<0.01$ versus $\mathrm{rV}$-LacZ-infected cells. 

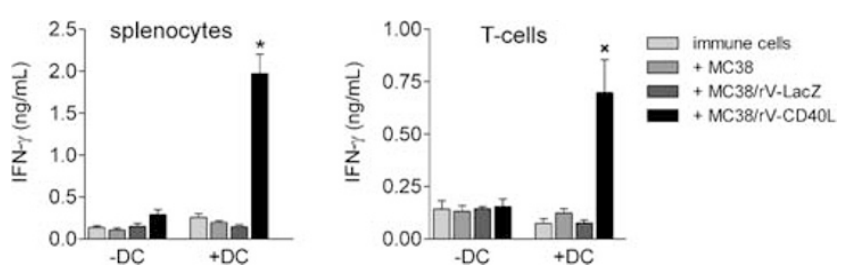

Figure 7 Analysis of IFN- $\gamma$ secretion by immune cells exposed to MC38 cells infected with rV-CD40L. Splenocytes or T cells were incubated for 48 hours with control MC38 cells or with MC38/rV$\mathrm{LacZ}^{\mathrm{R}}$ or $\mathrm{MC} 38 / \mathrm{rV}-\mathrm{CD}_{40 \mathrm{~L}^{\mathrm{R}}}$ in the presence of suboptimal doses of ConA ( $100 \mathrm{ng} / \mathrm{ml}$ for splenocytes and $200 \mathrm{ng} / \mathrm{ml}$ for T cells). Data are shown as the average $\pm S D$ of duplicate samples from one experiment representative of two performed. ${ }^{*} P<0.005 ;{ }^{\times} P<0.05$ versus rV-LacZ-infected cells.

near complete inhibition of proliferation, most probably due to a direct cytopathic effect of VV. Exposure of immune cells to MC38/rV-CD40L at the same MOI strongly stimulated proliferation of splenocytes and partially rescued T-cell proliferation (Fig 6e).

Despite the limited effect of MC38/rV-CD40L on T-cell proliferation in 48 hours in vitro cultures, strong stimulation of IFN- $\gamma$ secretion was observed by whole splenocyte cultures and purified $\mathrm{T}$ cells exposed to $\mathrm{MC} 38 / \mathrm{rV}-\mathrm{CD} 40 \mathrm{~L}$ (Fig 7). For T cells this effect was observed only in the presence of DC, suggesting that IL-12 released by stimulated DC contributed to the secretion of IFN- $\gamma$. The levels of IFN- $\gamma$ were 4- to 5-fold higher in both splenocyte and T-cell cultures after exposure to MC38/rV$\mathrm{CD} 4 \mathrm{~L}^{\mathrm{N}}$ compared to coincubation with $\mathrm{MC} 38 / \mathrm{rV}$ $\mathrm{CD}_{40 L^{\mathrm{R}}}$ (data not shown). MC38/rV-LacZ ${ }^{\mathrm{N}}$ did not influence IFN- $\gamma$ secretion. Interestingly, IL-4 levels were not elevated in any of the cocultures, suggesting that $\mathrm{MC} 38 / \mathrm{rV}-\mathrm{CD} 40 \mathrm{~L}$ can bypass T-cell help in the induction of B-cell proliferation (data not shown).

\section{$D X 5^{+}$(NK/NKT) cells proliferate and release IFN- $\gamma$ in response to MC38/rVCD4OL}

The high levels of IFN- $\gamma$ observed in splenocyte cultures exposed to $\mathrm{MC} 38 / \mathrm{rV}-\mathrm{CD} 40 \mathrm{~L}$ in the absence of $\mathrm{DC}$ prompted us to look for another CD40-expressing cell population that could release IFN- $\gamma$ upon direct stimulation with CD40L. Since naïve $\mathrm{CD}^{+}{ }^{+} \mathrm{T}$ cells are mostly CD40-negative, we focused on DX5 ${ }^{+} \mathrm{NK}$ and NKT cells, in which CD40L-dependent synthesis of IFN- $\gamma$ has been demonstrated. ${ }^{31}$ Flow cytometric analysis revealed that $\mathrm{DX} 5^{+}$cells could be divided into subpopulations based on the expression of different levels of NK1.1 and CD3, including two subpopulations of NKT cells that differ in the levels of CD3 expression (Fig 8a). Of these NKT cell populations that constituted about $18 \%$ of all DX5positive cells, the majority $(>50 \%)$ of $\mathrm{NK} 1.1^{+} \mathrm{CD} 3^{\text {lo }}$ cells and $33 \%$ of $\mathrm{NK}_{1.1}{ }^{+} \mathrm{CD} 3^{\text {hi }}$ cells expressed CD 40 , thus accounting for almost $90 \%$ of all CD40-expressing NKT cells. Thus, we evaluated the effect of MC38/rV$\mathrm{CD} 40 \mathrm{~L}$ on a DX5 ${ }^{+}$-enriched population and found that MC38/rV-CD40L strongly stimulated proliferation of a

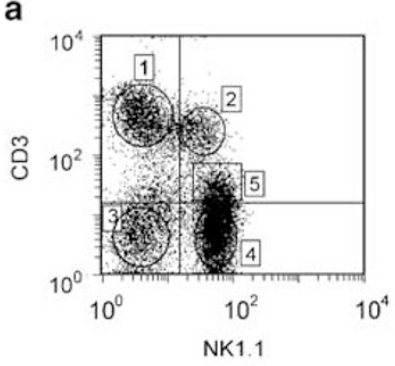

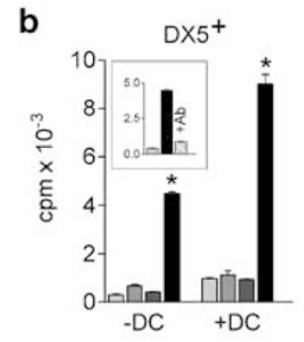

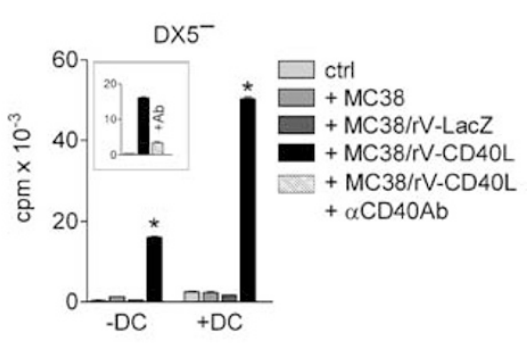

C
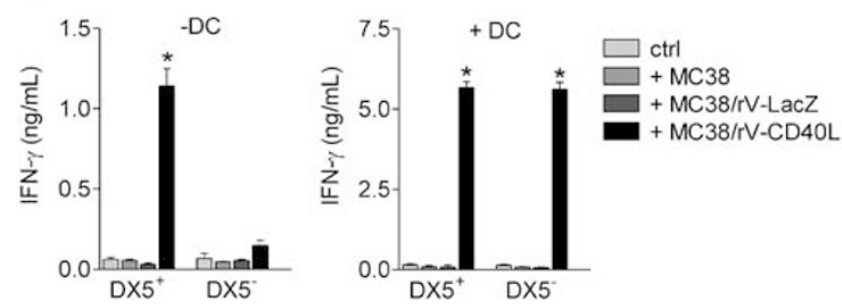

Figure 8 Analysis of $\mathrm{DX} 5^{+}$cell activation after exposure to MC38 cells infected with rV-CD40L. (a) Triple-color staining of $\mathrm{DX} 5^{+}$enriched splenocytes; (b) the incorporation of $\left[{ }^{3} \mathrm{H}\right]$-thymidine and $(\mathbf{c})$ IFN- $\gamma$ secretion by DX5 $5^{+}$and $\mathrm{DX} 5^{-}$cells incubated for 48 hours with control MC38, MC38/rV-LacZR or MC38/rV-CD40L ${ }^{\mathrm{R}}$ in the presence of ConA $(200 \mathrm{ng} / \mathrm{ml})$. Inhibition of proliferation by anti-CD40 polyclonal $A b$ in the absence of $D C$ was presented in the inserted graphs. Data are shown as the average \pm SD of duplicate samples from one experiment representative of two performed. ${ }^{*} P<0.01$ versus rV-LacZ infected cells.

DX $5^{+}$cells (Fig 8b). Furthermore, this effect was almost completely inhibited by anti-CD40 antibody $(100 \mu \mathrm{g} / \mathrm{ml})$ suggesting that $\mathrm{DX} 5^{+} \mathrm{CD} 40^{+}$cells proliferated through direct stimulation by CD40L on the surface of $\mathrm{MC} 38 / \mathrm{rV}$ CD40L. Since proliferation of $\mathrm{CD}^{+} \mathrm{T}$ cells was not increased by $\mathrm{MC} 38 / \mathrm{rV}-\mathrm{CD} 40 \mathrm{~L}$ and since $\mathrm{NK} 1.1^{+} \mathrm{CD} 3^{-}$ do not express CD40, the DX $5{ }^{+} \mathrm{NK} 1.1^{+} \mathrm{CD} 3{ }^{+} \mathrm{CD} 40^{+}$ NKT cells most likely constitute the responding cell population to direct $\mathrm{MC} 38 / \mathrm{rV}-\mathrm{CD} 40 \mathrm{~L}$ stimulation. This conclusion was further confirmed by evaluating IFN- $\gamma$ release in cocultures of DX $5^{+}$or $\mathrm{DX} 5^{-}$cells with MC38/rV-CD40L. Only DX5 ${ }^{+}$cells released significant amounts of IFN- $\gamma$ in response to MC38/rV-CD40L in the absence of DC (Fig 8c) and this cytokine release was abolished in the presence of anti-CD40 antibody (data not shown). However, similar to the results with purified $\mathrm{T}$ cells, DC provided efficient stimulation of IFN- $\gamma$ synthesis in the $\mathrm{DX} 5^{-}$population containing mostly $\mathrm{B}$ and $\mathrm{T}$ cells. These data support the conclusion that 
CD40L overexpression plays major role in direct stimulation of CD40-positive cells, including regulatory (NKT) and effector (B-cell) populations.

\section{Discussion}

A significant advantage of recombinant viral vectors has been the combination of adjuvant properties provided by the virus in the context of priming adaptive immunity through antigen expression from encoded transgenes. Recently, there has been a major focus on the coexpression of costimulatory molecules by poxviruses to further augment immune responses. ${ }^{20,21}$ The importance of CD40-CD40L interactions in priming $\mathrm{T}$-cell responses suggested that CD40L might be a therapeutically useful costimulatory molecule for increasing vaccine effectiveness, especially when designing antitumor vaccines against weakly immunogenic TAAs. Furthermore, efficient crosspresentation of TAAs by DC requires CD40CD40L "licencing" of DC. ${ }^{32}$ Thus, infection of tumor cells with rV-CD40L might serve a dual purpose. First, VV induces extensive cell death through both apoptotic and necrotic pathways, providing an abundance of cellular material for ingestion by DCs. ${ }^{24,33}$ Second, the expression of CD40L on tumor cells infected with $\mathrm{rV}$ CD40L further enhances TAA presentation by DCs, Despite this scenario, we found that the interaction of immune cells with rV-CD40L-infected tumor cells creates a complicated network of interactions with a high degree of unpredictability.

Our data provide additional insight into the understanding of some of the reported discrepancies regarding the role of CD40L in different experimental models. ${ }^{13,34}$ Although CD40L levels induced on $\mathrm{CD}^{+} \mathrm{T}$ cells following recognition of antigen is a rate-limiting factor in DC "licensing", subsequent interactions of CD40Lexpressing $\mathrm{CD}^{+}{ }^{+} \mathrm{T}$ cells with $\mathrm{B}$ cells and memory $\mathrm{CD} 8^{+}$ $\mathrm{T}$ cells are central to the network of cellular communication enabling efficient priming of immune responses and the generation of memory responses. ${ }^{35,36}$ Whereas CD40CD40L interactions are directly responsible for stimulation of IL-12 synthesis by DC, the downstream effects of IL-12 can be considered as an indirect effect of CD40CD40L interaction. In this context, CD40L on the surface of activated $\mathrm{CD}^{+}{ }^{+} \mathrm{T}$ cells directly stimulates $\mathrm{DC}$ and $\mathrm{B}$ cells that in turn generate signals in the form of costimulatory molecules or cytokines influencing $\mathrm{CD}^{+}$ $\mathrm{T}$ cells as well as other immune cells. We observed significant B-cell proliferation in cocultures with MC38/ rV-CD40L in the absence of DC (Fig 6b, d), without detectable IL-4 synthesis, indicating direct stimulation of B cells through CD40-CD40L interaction. Similar to B cells, stimulation of $\mathrm{DX} 5^{+}$cells with $\mathrm{MC} 38 / \mathrm{rV}-\mathrm{CD} 40 \mathrm{~L}$ was CD40 dependent since proliferation and IFN- $\gamma$ release were inhibited by anti-CD40 Ab in the absence of DC (Fig 8a and data not shown). The expression of CD40 on the surface of a DX $5^{+} \mathrm{NK} 1.1^{+} \mathrm{CD} 3^{\text {lo }}$ subpopulation of NKT cells, which has not been previously reported, raises the possibility that CD40-CD40L inter- action might be directly responsible for both NKT cell proliferation and IFN- $\gamma$ synthesis. However, it is also possible that VV may contribute additional stimulatory signals to these cells. Although IFN- $\gamma$ and IL-4 synthesis is a hallmark of Th1 and Th2 polarization, respectively, ${ }^{37}$ direct stimulation of B cells with CD40L leading to their proliferation in the presence of typical Th-1 cytokines indicates that indiscriminately overexpressed CD40L can override other factors involved in polarization of immune responses. Similarly, stimulation by CD40L might be directed toward important elements of cellular immunity due to the expression of CD40 on NKT-cells (our data) and $\mathrm{CD}^{+}{ }^{+}$memory $\mathrm{T}$ cells. ${ }^{35}$

Under in vivo conditions, intratumoral inoculation of $\mathrm{VV}$-based vectors will inevitably lead to infection of not only tumor cells but also DC at various levels of maturation. We found major differences in expression of virally encoded CD40L between DC and tumor cells (Fig 1). In fact, incubation of DC with tumor cells infected with rV-CD40L led to strong stimulation of IL12 synthesis while direct infection of DC with replicationcompetent rV-CD40L did not have this effect. This is consistent with another report demonstrating a diminished IL-12 response upon direct infection of DC with a recombinant VV expressing IL-2 produced in syngeneic cells. $^{38}$ This report suggested that DC were highly sensitive to the local environment and characteristics of the VV vectors used for infection. Using sucrose-purified viral particles, we observed stimulation of IL-12 synthesis by PS/UV-inactivated rV-CD40L, which was blocked by anti-CD40 Ab indicating that $\mathrm{rV}-\mathrm{CD} 40 \mathrm{~L}$ particles contained biologically active CD40L protein. This finding is not unexpected, particularly with recent evidence suggesting that intracellular expression of proteins from rVV transgenes often leads to incorporation of these proteins in both EEV and the more prevalent intracellular mature virion (IMV) particles. ${ }^{25,26}$ Moreover, it has been shown that proteins originating from host cells and carried by the virus might be biologically active as exemplified by retroviral vectors produced in FasL-expressing cell lines. ${ }^{39}$

The differences in IL-12 synthesis in DC exposed to replicating or nonreplicating rV-CD40L, or to MC38 cells infected with either of the viruses, indicate that the biologic effect of $\mathrm{rV}-\mathrm{CD} 40 \mathrm{~L}$ is difficult to predict and likely depends on the diverse effects of CD40L protein carried by the viral particles, intracellular expression of CD40L and the adjuvant properties as well as cytopathic effects of the viral vector. Further studies are required to elucidate the molecular mechanisms of this phenomenon. It is possible that employment of replication-defective vaccinia vectors, such as MVA, which ensures high levels of transgene expression and limited infectivity, might produce more predictable effects.

The manipulation of costimulatory activity for therapeutic purposes needs to consider the regulatory immune properties of the selected costimulatory molecule and the expression vector. The limitations of currently used viral vectors, including $\mathrm{rV}-\mathrm{CD} 40 \mathrm{~L}$ comprise the lack of proper targeting and timing of CD40L expression. Future vaccine development may be designed to take a full 
advantage of CD40-CD40L and other powerful costimulatory pathways. For example, placing CD40L under the control of a tissue-specific inducible promoter could prevent CD40L expression in infected DC, while ensuring overexpression in tumor cells, thus providing powerful signals for DC maturation and direct stimulation of antigen-specific immunity. Our results highlight the complexity of manipulating CD40-CD40L interaction with poxvirus-based vectors while supporting the feasibility of manipulating immune responses for therapeutic purposes.

\section{Acknowledgments}

We thank K Flanagan for assistance with virus preparation and culture. This work was supported by NIH Grant KO8 CA 79881 and the Committee for Scientific Research (KBN, 6 P04A 00120).

\section{References}

1. Hurwitz AA, Kwon ED, van Elsas A. Costimulatory wars: the tumor menace. Curr Opin Immunol. 2000;12:589-596.

2. Bromley SK, Iaboni A, Davis SJ, et al. The immunological synapse and CD28-CD80 interactions. Nat Immunol. 2001;2:1159-1166.

3. Creusot RJ, Mitchison NA, Terazzini NM. The immunological synapse. Mol Immunol. 2002;38:997-1002.

4. Grewal IS, Flavell RA. CD40 and CD154 in cell-mediated immunity. Annu Rev Immunol. 1998;16:111-135.

5. van Kooten C, Banchereau J. CD40-CD40 ligand. J Leukoc Biol. 2000;67:2-17.

6. Ye H, Arron JR, Lamothe B, et al. Distinct molecular mechanism for initiating TRAF6 signalling. Nature. 2002; 418:443-447.

7. Harte MT, Haga IR, Maloney G, et al. The poxvirus protein A52R targets Toll-like receptor signaling complexes to suppress host defense. J Exp Med. 2003;197:343-351.

8. Mackey MF, Wang Z, Eichelberg K, Germain RN. Distinct contributions of different CD40 TRAF binding sites to CD154-induced dendritic cell maturation and IL-12 secretion. Eur J Immunol. 2003;33:779-789.

9. Heufler C, Koch F, Stanzl U, et al. Interleukin-12 is produced by dendritic cells and mediates $\mathrm{T}$ helper 1 development as well as interferon-gamma production by $\mathrm{T}$ helper 1 cells. Eur J Immunol. 1996;26:659-668.

10. Tong AW, Stone MJ. Prospects for CD40-directed experimental therapy of human cancer. Cancer Gene Ther. 2003; 10:1-13.

11. Schonbeck U, Libby P. CD40 signaling and plaque instability. Circ Res. 2001;89:1092-1103.

12. Adler SH, Turka LA. Immunotherapy as a means to induce transplantation tolerance. Curr Opin Immunol. 2002; 14:660-665.

13. Homann D, Jahreis A, Wolfe T, et al. CD40L blockade prevents autoimmune diabetes by induction of bitypic NK/ DC regulatory cells. Immunity. 2002;16:403-415.

14. Perez-Melgosa M, Hollenbaugh D, Wilson CB. Cutting edge: CD40 ligand is a limiting factor in the humoral response to T cell-dependent antigens. J Immunol. 1999;163: $1123-1127$.
15. Ahlers JD, Belyakov IM, Thomas EK, Berzofsky JA. Highaffinity $\mathrm{T}$ helper epitope induces complementary helper and APC polarization, increased CTL, and protection against viral infection. $J$ Clin Invest. 2001;108:1677-1685.

16. Grassme H, Bock J, Kun J, Gulbins E. Clustering of CD40 ligand is required to form a functional contact with CD40. J Biol Chem. 2002;277:30289-30299.

17. Ruby J, Bluethmann H, Aguet M, Ramshaw IA. CD40 ligand has potent antiviral activity. Nat Med. 1995;1:437-441.

18. Kato K, Cantwell MJ, Sharma S, Kipps TJ. Gene transfer of CD40-ligand induces autologous immune recognition of chronic lymphocytic leukemia B cells. J Clin Invest. 1998; 101:1133-1141

19. Wendtner CM, Kofler DM, Theiss HD, et al. Efficient gene transfer of CD40 ligand into primary B-CLL cells using recombinant adeno-associated virus (rAAV) vectors. Blood. 2002;100:1655-1661.

20. Rolph MS, Ramshaw IA. Recombinant viruses as vaccines and immunological tools. Curr Opin Immunol. 1997;9: $517-524$

21. Hodge JW, Grosenbach DW, Schlom J. Vector-based delivery of tumor-associated antigens and T-cell co-stimulatory molecules in the induction of immune responses and anti- tumor immunity. Cancer Detect Prev. 2002;26: 275-291.

22. Hodge JW, Sabzevari H, Yafal AG, Gritz L, Lorenz MG, Schlom J. A triad of costimulatory molecules synergize to amplify T-cell activation. Cancer Res. 1999;59: 5800-5807.

23. Yewdell JW, Hill AB. Viral interference with antigen presentation. Nat Immunol. 2002;3:1019-1025.

24. Larsson M, Fonteneau JF, Somersan S, et al. Efficiency of cross presentation of vaccinia virus-derived antigens by human dendritic cells. Eur J Immunol. 2001;31:3432-3442.

25. Gomez CE, Esteban M. Recombinant proteins produced by vaccinia virus vectors can be incorporated within the virion (IMV form) into different compartments. Arch Virol. 2001;146:875-892.

26. Krauss O, Hollinshead R, Hollinshead M, Smith GL. An investigation of incorporation of cellular antigens into vaccinia virus particles. J Gen Virol. 2002;83:2347-2359.

27. Broder CC, Earl PL. Design and construction of recombinant vaccinia viruses. Methods Mol Biol. 1997;62:173-197.

28. Inaba $\mathrm{K}$, Inaba $\mathrm{M}$, Romani $\mathrm{N}$, et al. Generation of large numbers of dendritic cells from mouse bone marrow cultures supplemented with granulocyte/macrophage colony-stimulating factor. $J$ Exp Med. 1992;176:1693-1702.

29. Tsung K, Yim JH, Marti W, Buller RM, Norton JA. Gene expression and cytopathic effect of vaccinia virus inactivated by psoralen and long-wave UV light. $J$ Virol. 1996;70:165-171.

30. Ramsey-Ewing A, Moss B. Apoptosis induced by a postbinding step of vaccinia virus entry into Chinese hamster ovary cells. Virology. 1998;242:138-149.

31. Hayakawa Y, Takeda K, Yagita H, Van Kaer L, Saiki I, Okumura K. Differential regulation of Th1 and Th2 functions of NKT cells by CD28 and CD40 costimulatory pathways. J Immunol. 2001;166:6012-6018.

32. Schoenberger SP, Toes RE, van der Voort EI, Offringa R, Melief CJ. T-cell help for cytotoxic T lymphocytes is mediated by CD40-CD40L interactions. Nature. 1998;393:480-483.

33. Cho HJ, Bhardwaj N. Against the self: dendritic cells versus cancer. Apmis. 2003;111:805-817.

34. French RR, Chan HT, Tutt AL, Glennie MJ. CD40 antibody evokes a cytotoxic T-cell response that eradicates lymphoma and bypasses T-cell help. Nat Med. 1999;5:548-553. 
35. Bourgeois C, Rocha B, Tanchot C. A role for CD40 expression on CD8 $+\mathrm{T}$ cells in the generation of CD $8+\mathrm{T}$ cell memory. Science. 2002;297:2060-2063.

36. Dubois B, Vanbervliet B, Fayette J, et al. Dendritic cells enhance growth and differentiation of CD40-activated B lymphocytes. J Exp Med. 1997;185:941-951.

37. Spellberg B, Edwards Jr JE. Type 1/Type 2 immunity in infectious diseases. Clin Infect Dis. 2001;32:76-102.
38. Mukherjee S, Upham JW, Ramshaw I, et al. Dendritic cells infected with a vaccinia virus interleukin-2 vector secrete high levels of IL-2 and can become efficient antigen presenting cells that secrete high levels of the immunostimulatory cytokine IL-12. Cancer Gene Ther. 2003;10: 591-602.

39. Jodo S, Strehlow D, Ju ST. Bioactivities of Fas ligandexpressing retroviral particles. J Immunol. 2000;164:5062-5069. 